\title{
Light-Driven Raman Coherence as a Nonthermal Route to Ultrafast Topology Switching in a Dirac Semimetal
}

\author{
C. Vaswani, ${ }^{1}$ L.-L. Wang, ${ }^{1}$ D. H. Mudiyanselage, ${ }^{1}$ Q. Li$^{2},{ }^{2}$ P. M. Lozano, ${ }^{2}$ G. D. Gu, ${ }^{2}$ D. Cheng, ${ }^{1}$ B. Song, ${ }^{1}$ \\ L. Luo, ${ }^{1}$ R. H. J. Kim $\odot,{ }^{1}$ C. Huang, ${ }^{1}$ Z. Liu, ${ }^{1}$ M. Mootz, ${ }^{3}$ I. E. Perakis $\odot,{ }^{3}$ Y. Yao $\odot,{ }^{1}$ K. M. Ho, ${ }^{1}$ and J. Wang $\oplus^{1, *}$ \\ ${ }^{1}$ Department of Physics and Astronomy and Ames Laboratory, U.S. Department of Energy, \\ Iowa State University, Ames, Iowa 50011, USA \\ ${ }^{2}$ Condensed Matter Physics and Materials Sciences Department, Brookhaven National Laboratory, \\ Upton, New York 11973-5000, USA \\ ${ }^{3}$ Department of Physics, University of Alabama at Birmingham, Birmingham, Alabama 35294-1170, USA
}

(Received 21 November 2019; revised manuscript received 30 January 2020; accepted 5 March 2020; published 17 April 2020)

\begin{abstract}
A grand challenge underlies the entire field of topology-enabled quantum logic and information science: how to establish topological control principles driven by quantum coherence and understand the time dependence of such periodic driving. Here we demonstrate a few-cycle THz-pulse-induced phase transition in a Dirac semimetal $\mathrm{ZrTe}_{5}$ that is periodically driven by vibrational coherence due to excitation of the lowest Raman active mode. Above a critical THz-pump field threshold, there emerges a long-lived metastable phase, approximately 100 ps, with unique Raman phonon-assisted topological switching dynamics absent for optical pumping. The switching also manifests itself by distinct features: nonthermal spectral shape, relaxation slowing near the Lifshitz transition where the critical Dirac point occurs, and diminishing signals at the same temperature that the Berry-curvature-induced anomalous Hall effect magnetoresistance vanishes. These results, together with first-principles modeling, identify a modeselective Raman coupling that drives the system from strong to weak topological insulators with a Dirac semimetal phase established at a critical atomic displacement controlled by the phonon coherent pumping. Harnessing of vibrational coherence can be extended to steer symmetry-breaking transitions, i.e., Dirac to Weyl ones, with implications for $\mathrm{THz}$ topological quantum gate and error correction applications.
\end{abstract}

DOI: 10.1103/PhysRevX.10.021013

Subject Areas: Condensed Matter Physics, Optics

\section{INTRODUCTION}

Dynamic driving by periodic lattice vibrations represents a powerful approach to manipulate topological band structures, in stark contrast to equilibrium tuning methods, e.g., temperature, chemical substitution, and static strain, electric, or magnetic fields [1,2]. Ultrafast nonthermal manipulation of topology $[3,4]$, particularly at preferred $\mathrm{THz}$-cycle clock rates, is key for full implementation of the dynamical protocols needed to both match current information and sensing technologies and exceed their limits via topological functionalities [5-8]. Despite the recent intriguing studies of coherent phonon pumping in various quantum materials [9-12], topological semimetals dressed by optical phonon coherence have not been experimentally

\footnotetext{
* Corresponding author. jwang@ameslab.gov

Published by the American Physical Society under the terms of the Creative Commons Attribution 4.0 International license. Further distribution of this work must maintain attribution to the author(s) and the published article's title, journal citation, and DOI.
}

explored, especially for Dirac materials. $\mathrm{ZrTe}_{5}$ is a model Dirac system [13-15] for studying such a quantum topological switching by periodic driving from coherent phonons because of the minimal single nodal (Dirac) point and extreme sensitivity on the small structural changes across a broad range of phases, from strong topological insulators (STIs) to Dirac semimetal to weak topological insulators (WTIs). However, only thermal- or strain-induced transitions $[16,17]$ in quasiequilibrium states have been reported.

We implement a dynamical topology-switching scheme using intense few-cycle $\mathrm{THz}$ pulses (red line) to excite a Raman active $\left(A_{1 g}\right)$ optical phonon mode, as illustrated in Fig. 1(a). The subpicosecond $\mathrm{THz}$ driving has broadband spectrum with central frequency of approximately $1.2 \mathrm{THz}$ [gray shaded area, Fig. 1(b)]. The $A_{1 g}$ eigenmode [Fig. 1(c)] is a translational rigid-chain structure which mostly involves opposite displacements along the $b$ axis of the dimer $\mathrm{Te}\left(\mathrm{Te}_{d}\right)$, apical $\mathrm{Te}\left(\mathrm{Te}_{a}\right)$, and $\mathrm{Zr}$ atoms from the neighboring $\mathrm{Zr}$-Te units. This motion results in a modulation of the atomic positions along the $b$ axis that determines the van der Waals coupling and, in turn, controls the band inversion at the $\Gamma$ point between the $p$ orbitals of $\mathrm{Te}_{d}$ and zigzag $\mathrm{Te}_{z}$ atoms. Most intriguingly, this mode 

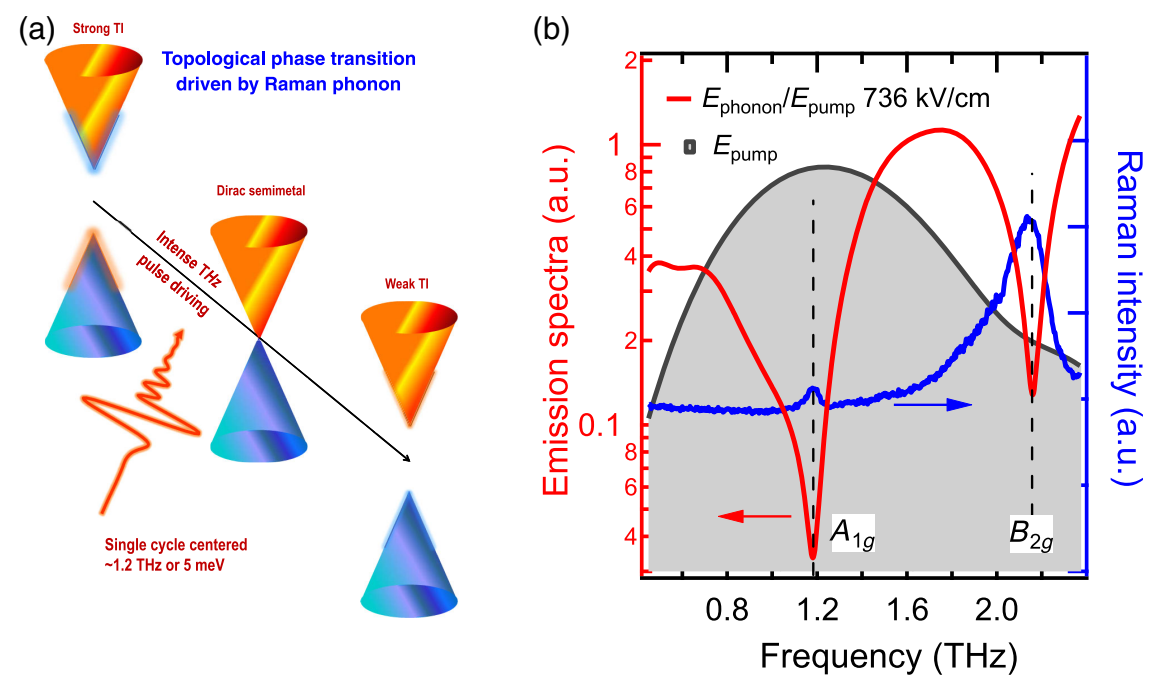

(c)

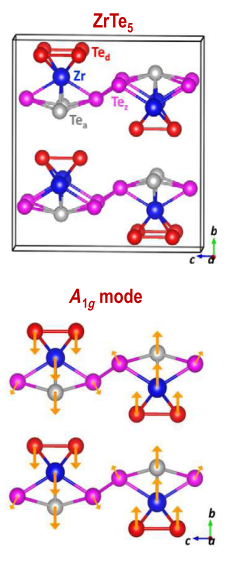

(d)

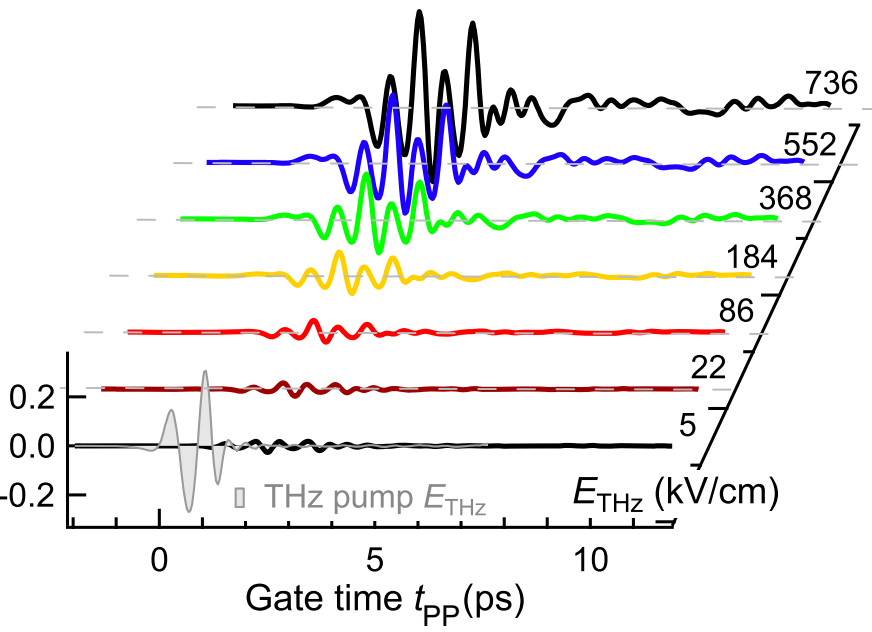

FIG. 1. Intense THz-driven Raman phonon coherence in $\mathrm{ZrTe}_{5}$. (a) Schematic of the topological switching driven by the THz coherent excitation in Dirac semimetals. (b) Spectrum of the coherent phonon emission (red) obtained from the time-domain traces shown for maximum THz-field strengths $E_{\max }=736 \mathrm{kV} / \mathrm{cm}$. The pump THz spectrum is shown in gray. Dashed lines indicate the identification of the excited modes in the driven states with the measured Raman spectrum (blue). (c) Crystal structure of $\mathrm{ZrTe}_{5}$ and the relative atomic displacements of the $A_{1 g}$ Raman mode leading to the modulation of interlayer spacing. The arrows indicate the direction and magnitude of the atomic displacements. (d) Time-domain traces of the measured coherent phonon emission for various THz-field strengths. The THz-driving pulse is shown in gray.

could effectively enable a topology change without symmetry breaking because it has extreme sensitivity to the band inversion while it still preserves the inversion symmetry of the lattice. Coherent excitation of the $A_{1 g}$ phonon is expected to create a periodically driven state via lattice vibrations that modulate the topological bands and switch from STI [top, Fig. 1(a)] to Dirac semimetal (middle) to WTI (bottom) phases. Dephasing of this topological coherent state via Dirac fermion-phonon interaction leads to the conversion of Raman phonon coherence into population, i.e., into finite atomic displacement associated with the establishment of a final state with highly nonthermal characteristics. However, the salient spectroscopy features for the ultrafast nonthermal phase transition in any Dirac materials have not been established until now.
In this paper, we provide evidence for a distinct topology switching driven by $\mathrm{THz}$ Raman phonon coherence in a Dirac semimetal. Our results are corroborated by the calculations of $A_{1 g}$ mode-selective electronic band structures and topology changes. Harnessing the driven coherence of specifically tailored phonon modes with an intense near-single-cycle $\mathrm{THz}$ pulse may become a universal principle for steering topological phase transitions.

\section{EXPERIMENTAL METHODS AND RESULTS}

We use a bulk single-crystal $\mathrm{ZrTe}_{5}$ sample that exhibits a 3D linear dispersion [18]. In equilibrium, our sample is close to charge neutral, and the Fermi level $E_{F}$ is less than $5 \mathrm{meV}$ and approximately $14 \mathrm{meV}$ at $5 \mathrm{~K}$ and room 
temperature, respectively. It has been reported that $E_{F}$ is proportional to the Lifshitz transition temperature $T_{\text {Lifshitz }}$ that depends on the Te content [19]. $\mathrm{ZrTe}_{5}$ single crystals grown by Te self-flux, as we use, are close to the stoichiometric value and have lower $E_{F}$ and $T_{\text {Lifshitz, }}$, while those crystals grown by the chemical-vapor-transport method are sometimes Te deficient and have higher values. Intense near-single-cycle THz-pump pulses are generated through optical rectification employing the tilted pulse front scheme in $\mathrm{LiNbO}_{3}$ using 800-nm 35-fs pulses from a 1-kHz Ti:sapphire regenerative amplifier system. A detailed description of the THz-pump, THz-probe, and $\mathrm{THz}-\mathrm{emission}$ setups and data acquisition scheme can be found in Refs. [8,20-23].

Coherent phonon emissions after intense THz-pump excitation at $4.1 \mathrm{~K}$ for various field strengths $E_{\mathrm{THz}}=5$, $22,86,184,386,552$, and $736 \mathrm{kV} \mathrm{cm}^{-1}$ are sampled in the time domain by a weak optical pulse, as shown in Fig. 1(d). A pronounced multicycle oscillation is clearly visible in the sample emission. The Fourier-transform spectra of these coherent beatings at $736 \mathrm{kV} \mathrm{cm}^{-1}$ display two dominant peaks centered at approximately 1.2 and $2.1 \mathrm{THz}$. The static Raman spectrum [blue line, Fig. 1(b)] from the same sample shown together, identifies their Raman symmetry (dash lines). Note that the strongest emission peak of approximately $1.2 \mathrm{THz}$ in the driven states matches very well with the $A_{1 g}$ mode, unlike for the $B_{2 g}$ mode dominant in the static Raman spectra. Thus, the intense $\mathrm{THz}$ drive coherently excites the Raman $A_{1 g}$ mode resulting in a periodic modulation of the interlayer spacing. In addition, the three infrared (IR) active phonon modes with frequencies $0.63,1.5$, and $2.3 \mathrm{THz}$, as seen in linear THz transmission (see Fig. S2 in the Supplemental Material [24]) are negligibly small in the intense THz-driven state [Fig. 1(d)], i.e., the preferred coupling of the topological electronic bands to the $A_{1 g}$ mode. This Raman-mode-selective coupling attests the proposed excitation scheme in Fig. 1(c) due to the extreme sensitivity of the band inversion to the interlayer spacing, which is reproduced by densityfunctional simulations that we discuss later.

To characterize the observed periodically driven topological state dressed by Raman coherence, Fig. 2(a) plots the $\mathrm{THz}$ differential transmission $\Delta E / E_{0}$ (red circles) after excitation by the intense THz-pump pulse (gray shaded area) as a function of the pump-probe time delay $\Delta t_{\mathrm{PP}}$ at $4.1 \mathrm{~K}$. Note that the pump-induced signals are normalized by the value of peak amplitude $E_{0}$ at the probe $\mathrm{THz} E$ field (gray shaded area, inset). The nonequilibrium signal $\Delta E / E_{0}$ measured at the gating pulse delay $t_{\mathrm{gate}}=0 \mathrm{ps}$ (inset) originates from multiple processes ranging from intra- and interband excitations assisted by coherent phonons, to phonon renormalization and recovery of the quasiequilibrium Dirac state, e.g., via scattering within and between the Dirac cones via electron-electron $(e-e)$ and electron-phonon (e-ph) interactions. The
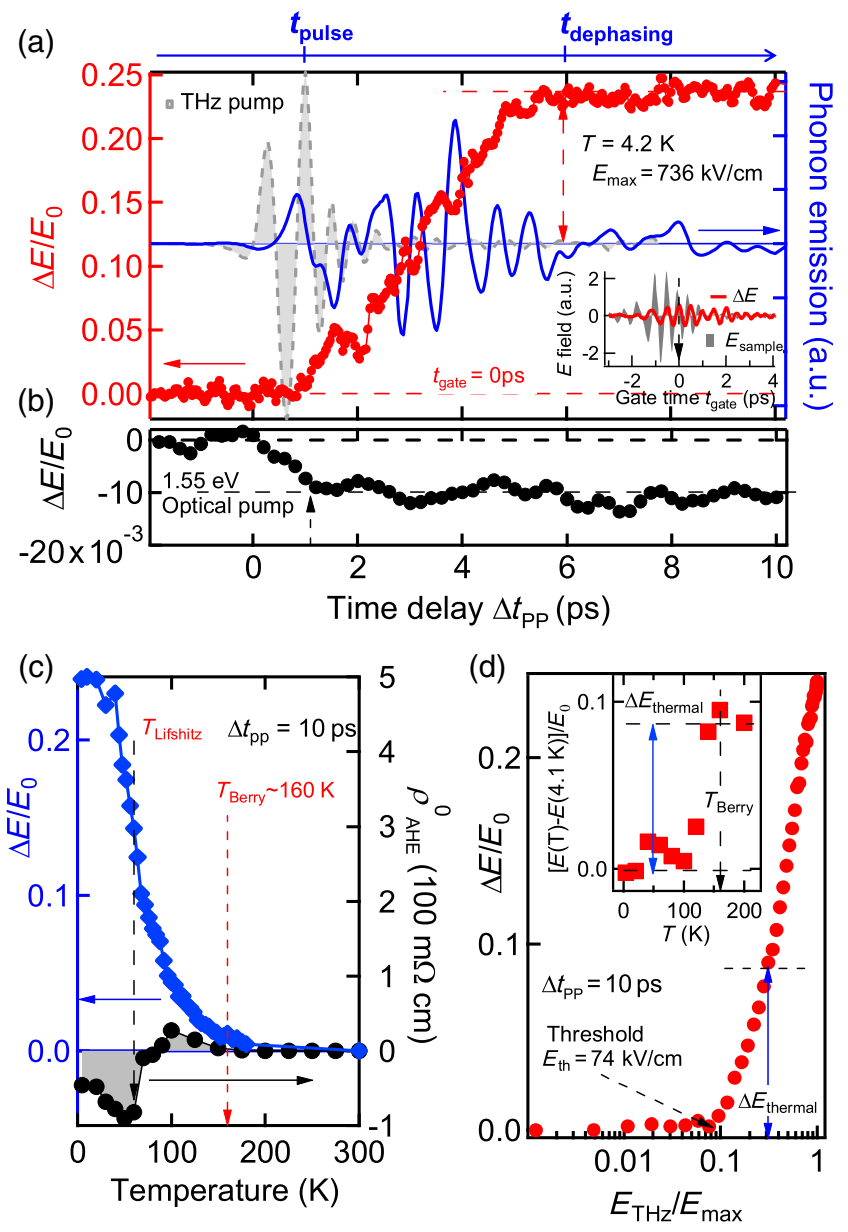

FIG. 2. Light-driven topological phase transition above threshold THz-pump field and at low temperature where the Berry curvature dominates. (a) $\mathrm{THz}$ differential transmission $\left(\Delta E / E_{0}\right.$, red circles) as a function of the pump-probe time delay $\left(\Delta t_{\mathrm{PP}}\right)$ for a peak pump $E$ field of $736 \mathrm{kV} / \mathrm{cm}$. The blue trace shows the simultaneously measured phonon emission. The THz-pump trace is shown in gray. The $\mathrm{THz}$ differential transmission starts to build up during the coherent phonon emission at much longer times than the pump $\mathrm{THz}$ pulse. Inset: Time-domain $\mathrm{THz}$ raw data. The transmitted probe $E$ field through the sample $E_{\text {sample }}$ (gray shaded area) and the pump-induced transmitted probe $E$-field change $\Delta E$ (red line) at $\Delta t_{\mathrm{PP}}=10 \mathrm{ps}$ are measured by scanning $t_{\text {gate }}$. We normalize the pump-induced signals by the value of peak amplitude $E_{0}$ at the probe $\mathrm{THz} E$ field. (b) $\mathrm{THz}$ differential transmission for the case of high-energy optical pump (1.55 eV) shows a much faster rise time. (c) Temperature dependence of the differential $\mathrm{THz}$ transmission in the metastable state $\left(\Delta t_{\mathrm{PP}}=10 \mathrm{ps}\right.$, blue diamonds) indicates strong correlation with the Berry-curvature-induced anomalous Hall effect (black solid circles). (d) Pump E-field dependence of the THz differential transmission for the $\mathrm{THz}$ pump at $\left(\Delta t_{\mathrm{PP}}=10 \mathrm{ps}\right)$, indicating a threshold pump field for the formation of the metastable state. Inset: The thermal topological change $\Delta E_{\text {thermal }}$ required for the thermally driven STI-DP-WTI transition is determined by the change of THz-field transmission between 4.1 and $160 \mathrm{~K}\left(T_{\text {Berry }}\right)$. 
dynamics is further elaborated later. For clarity, shown together is the pump-induced coherent phonon emission (black line, overlaid) that is measured simultaneously as a function of $\Delta t_{\mathrm{PP}}$. These results reveal the buildup of a metastable state, which occurs exclusively during the coherent Raman phonon oscillations in time domain. At the early times during the THz pulse marked by $t_{\text {pulse }}$ [Fig. 2(a)], there is only small pump-induced $\Delta E / E_{0}$ signals. The delayed rise in ultrafast transient signals excludes the direct heating of electronic states near the Fermi surface as the dominant excitation channel, which will lead to quasi-instantaneous increase in the transient signals on the arrival of the pump pulse. In contrast, the transient-state evolution seen from the pronounced $\Delta E / E_{0}$ signals occurs only at later times after the pulse but before phonon dephasing marked as $t_{\text {dephasing, }}$ i.e., during the period of the pronounced coherent phonon vibrations [blue line, Fig. 2(a)]. This emergent behavior after the incident $\mathrm{THz}$ excitation dominates the driven-state dynamics. The formation process is followed by a quasisteady temporal regime that marks the establishment of a final metastable state after the dephasing of the Raman phonon coherence by, e.g., the strong $e-e$ and $e$-ph coupling. In addition, the buildup behavior during coherent oscillations is absent for high-frequency pump pulses tuned at $1.55 \mathrm{eV}$ [Fig. 2(b)]. Here we see only a sub-ps rise which now occurs mostly during the photoexcitation. This stark difference clearly indicates the nonthermal nature of the THz-driven state mediated by coherent Raman phonons.

Experimental evidence associating the observed phase evolution with the topological switching is presented in Figs. 2(c) and 2(d) as follows. The first evidence is to compare the temperature dependence of the pump-probe $\Delta E / E_{0}$ signals in the metastable states (at $\Delta t_{\mathrm{PP}}=10 \mathrm{ps}$ ) with that of the anomalous Hall effect (AHE) magnetoresistance that directly probes the Berry curvature $\Omega_{k}$ generated by the Weyl nodes. As shown in Fig. 2(c), the nonlinear $\mathrm{THz}$ signal (blue diamond) quickly diminishes at the same temperature $T_{\text {Berry }} \sim 160 \mathrm{~K}$, where AHE sensitivity $\rho_{\mathrm{AHE}}^{0}$ vanishes (black circles). In the AHE measurement, application of a magnetic field transforms a Dirac semimetal into a Weyl semimetal by breaking time-reversal symmetry. Consequently, Weyl nodes behave like magnetic monopoles that generate large Berry curvatures and act like an effective magnetic field. This field-induced Berry curvature gives rise to a nonzero $\rho_{\text {AHE }}=\Omega_{k} \times E$. Here we obtain the temperature dependence of $\rho_{\mathrm{AHE}}^{0}$, i.e., the saturation value of $\rho_{\mathrm{AHE}}(B)$ (see Fig. S1 in the Supplemental Material [24]) by subtracting the ordinary Hall signals (linear background) at high magnetic field from the experimentally measured Hall resistivity [25]. It is clearly visible that $\rho_{\mathrm{AHE}}^{0}$ in $\mathrm{ZrTe}_{5}$ emerges below $T_{\text {Berry }}$ when the dominant carriers are Dirac fermions with linear dispersion near the conical point with conserved chirality. The $T_{\text {Berry }}$ correlates very well with the critical transition temperature associated with the THz-driven metastable phase [blue diamond, Fig. 2(c)]. Therefore, the metastable phase has the same topological origin as the chiral transport and cannot be established by excitation of a normal Fermi surface dominant above $T_{\text {Berry }}$. Note also that the sign change of $\rho_{\mathrm{AHE}}^{0}$ in the vicinity of $T_{\text {Lifshitz }} \sim 60 \mathrm{~K}$ where the critical DP occurs separating the STI-WTI transitions [16] [see Fig. S1(b) in the Supplemental Material [24] ] agrees with the rapid rise of the THz-pump-induced signals in the driven states marked by the black dashed line associated with the buildup of the new phase.

The second evidence is a distinct nonlinear pump fluence dependence of the $\mathrm{THz}$ differential transmission $\Delta E / E_{0}$. It exhibits both photoexcitation threshold behavior and larger magnitude than the change required for the thermally driven topological phase transition denoted as $\Delta E_{\text {thermal }}$. Specifically, by increasing the temperature from 4.2 to $160 \mathrm{~K}$ ( $\left.T_{\text {Berry }}\right)$, as we show in the inset of Fig. 2(d), the $\Delta E_{\text {thermal }}$ can be directly determined as approximately 0.09 corresponding to the change of THzfield transmission during the STI-DP-WTI transition in equilibrium. Here we compare the $\Delta E_{\text {thermal }}$ with the THz-pump field dependence of the differential transmission $\Delta E / E_{0}$ signals at a fixed time $\Delta t_{\mathrm{PP}}=10 \mathrm{ps}$ [Fig. 2(d)]. We emphasize two key points. First, it is clearly visible that the pump-induced $\Delta E / E_{0}$ is negligibly small at $\mathrm{THz}$-field strengths less than $E_{\mathrm{th}} \sim 75 \mathrm{kV} / \mathrm{cm}$. This threshold behavior of the formation dynamics [Fig. 2(a)] is not limited by our noise floor, which is a hallmark of the nonequilibrium phase transition to a THz-driven metastable state. Second, at slightly higher field above the $E_{\mathrm{th}}$, the pump-induced differential transmission surpasses the $\Delta E_{\text {thermal }}$ value for the thermally induced STI-DP-WTI topological switching in equilibrium (black dashed line). Thus, we conclude that the sufficiently large lattice displacement above the $E_{\text {th }}$ drives the system across the topological phase boundary to new band structures determined by phonon vibration amplitudes.

Next, we further identify some distinguishing spectral and temporal features associated with the $\mathrm{THz}$-driven metastable topological phase that are different from the thermal states. First, Fig. 3(a) reveals a distinct nonthermal spectral shape in the nonequilibrium response function. At equilibrium, the frequency-dependent conductivity $\sigma_{1} / \sigma_{\mathrm{dc}}$ [inset, Fig. 3(a)] reveals two strong IR-active phonons along the $a$ axis (probe direction) with resonant peaks at $\omega_{\mathrm{IR}}^{1,2} \sim 1.5$ and approximately $2.5 \mathrm{THz}$ in the $4.2-\mathrm{K}$ trace (red line). These IR phonon modes can contribute to the Raman $A_{1 g}$ phonon generation via the ionic Raman mechanism that involves the IR modes as a mediator and IR Raman coupling due to anharmonicity [26]. At elevated temperatures, these modes progressively shift to higher frequencies up to $200 \mathrm{~K}$ (magenta line). In the 

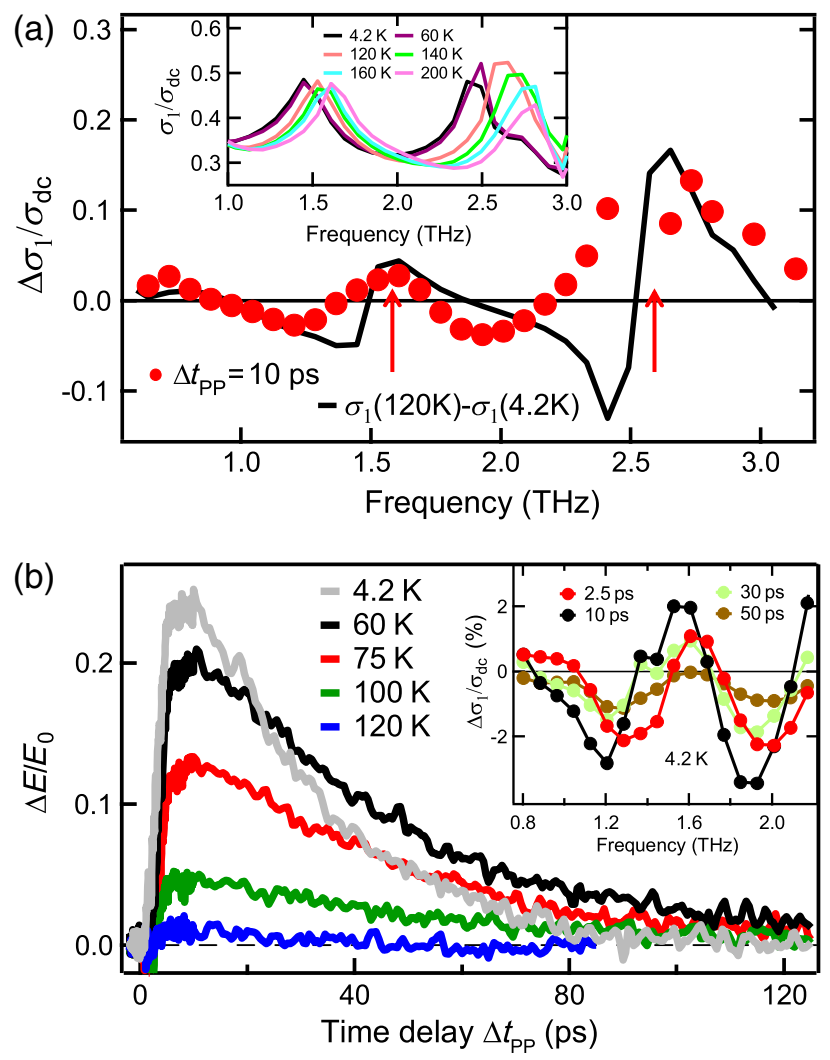

FIG. 3. The distinct spectral and temporal features of the highly nonthermal, metastable topological states. (a) Pumpinduced change in conductivity $\left(\Delta \sigma_{1} / \sigma_{\mathrm{dc}}\right.$, red circles) at $\Delta t_{\mathrm{PP}}=$ $10 \mathrm{ps}$ and $T=4.2 \mathrm{~K}$ compared with the thermal conductivity change (black circles) between 120 and $4.2 \mathrm{~K}$, i.e., STI and WTI states. Inset shows the shift in the IR phonon frequencies in the static conductivity along the $a$ axis as a function of the temperature. (b) Differential $\mathrm{THz}$ transmission profiles $\left(\Delta E / E_{0}\right)$ for various temperatures show that the relaxation of the metastable state is the longest near $T_{\text {Lifshitz }} \sim 60 \mathrm{~K}$. Inset: $\mathrm{THz}$ response $\Delta \sigma_{1} / \sigma_{\mathrm{dc}}$ as a function of the frequency for time delays $\Delta t_{\mathrm{PP}}=2.5,10,30$, and $50 \mathrm{ps}$ at $E_{\mathrm{THz}}=736 \mathrm{kV} / \mathrm{cm}$ at $4.2 \mathrm{~K}$.

THz-driven phase, the pump-induced conductivity change $\Delta \sigma_{1} / \sigma_{\mathrm{dc}}$ at $\Delta t_{\mathrm{PP}}=10 \mathrm{ps}$ shows spectral oscillations [red circles, Fig. 3(a)] with pronounced absorptive features (red arrows). In contrast, the normal-state thermalization leads to dominantly inductive spectral shape [black line, Fig. 3(a)], which can be obtained by subtracting $\sigma_{1}$ traces at higher temperature and $4.2 \mathrm{~K}$, i.e., between the STI ground and WTI thermal states. This result highlights the difference between the driven phase evolution and temperatureor laser-heating-induced phase thermalization process. Second, Fig. 3(b) plots the relaxation dynamics that measures the lifetime of the THz-driven state from 4.2 to $120 \mathrm{~K}$. The temporal profile is consistent with the slow buildup peaked at approximately $10 \mathrm{ps,} \mathrm{and} \mathrm{approximately}$ 100 -ps decay of transient Dirac fermion population and phonon renormalization, as seen in the $\sigma_{1} / \sigma_{\mathrm{dc}}$ spectra shown for various $\Delta t_{\mathrm{PP}}$ delays [inset, Fig. 3(b)]. The transient phase decays with a single exponential profile over approximately $120 \mathrm{ps}$; see, e.g., the $60-\mathrm{K}$ trace (black line). Such a relaxation is nearly 2 orders of magnitude longer than that reported for the case of optical excitation using higher-energy photons [27,28]. This observation is due in part to the minimal electronic or phonon heating and negligible excitation of other topological trivial bands from the low-frequency $\mathrm{THz}$ pumping. Intriguingly, the relaxation time exhibits a nonmonotonic temperature dependence. It becomes longer initially with temperature increase from $4.2 \mathrm{~K}$ (gray line), reaches a maximum at approximately $60 \mathrm{~K}$ (black line), and finally decreases with a temperature increase up to $120 \mathrm{~K}$ (blue line), as shown in Fig. 3(b). Critical to note is that the longest lifetime appears at approximately $T_{\text {Lifshitz }}$ at which the Fermi surface crosses the Dirac point [16]. The prolonged decay times and their correlation with the Lifshitz transition are exceptional in $\mathrm{ZrTe}_{5}$, which reflects the unique dynamics of Dirac bands, instead of other topologically trivial ones. This correlation further underscores the topological origin of the THz-driven phase transition as we discuss below.

Coherent and nonthermal $\mathrm{THz}$ photoexcitations alter the thermodynamic equilibrium of electronic states and lead to a series of dynamic processes arising from the generation and decay of transient Dirac fermion and phonon population in $\mathrm{ZrTe}_{5}$. During and immediately following the single-cycle $\mathrm{THz}$ excitation, strongly driven coherent phonons modulate the Dirac bands and decrease the gap size and Fermi energy. These periodic gap closing by coherent phonons assist the interband excitation between the Dirac cones and the intraband Fermi sea pumping. The excitation is followed by buildup of a quasiequilibrium Dirac state after the dephasing. Then, for longer times this quasiequilibrium Dirac state relaxes via scattering within and between the Dirac cones via intraband and interband transitions. These processes determine the decay dynamics and slowing behavior observed in Fig. 3(b). We emphasize two key points. First, the linear Dirac spectrum gives rise to slow imbalance relaxation. As it is rigorously formulated in graphene, the number of transient electrons and holes in the upper and lower Dirac bands are individually conserved up to second order in the Coulomb interaction [29]. Second, near the Lifshitz transition when the Fermi energy approaches the conical point, there will be further slowing in the decay due to kinematic phase-space constraints imposed on the relaxation process: less scattering due to reduced density of states between the Dirac cones (interband) and within them (intraband) via phonons. Note that our observed slow decay is consistent with similar "tens of" for clarity of ps lifetimes reported in carrier-density-tuned topological insulators [30] and should be distinguished from the dynamic slowing mechanism in symmetry-breaking phase transitions [31]. 


\section{ELECTRONIC STRUCTURE CALCULATIONS AND DISCUSSIONS}

To put the observed Raman-mode-selective topological phase transition on stronger footing, we carry out phonon and electronic band structure calculations based on densityfunctional theory (DFT) that provide theoretical support for the topological switching by the coherent $A_{1 g}$ phonon driving. Among the calculated zone-center optical phonon modes with the relaxed unit cell (see Supplemental Material [24]), the lowest Raman $A_{1 g}$ mode of $1.12 \mathrm{THz}$ and $B_{2 g}$ mode of $2.18 \mathrm{THz}$ compare well with the experimental values [32] of 1.18 and $2.16 \mathrm{THz}$, respectively. To study the effect of this $A_{1 g}$ mode on the electronic structure and band topology of $\mathrm{ZrTe}_{5}$, we change the atomic positions according to the $A_{1 g}$ mode [see Fig. 1(c)] with a lattice distortion factor $(\lambda)$ and calculate the new band structure. For $\lambda=1.0$, the corresponding atomic displacements are $0.033 \AA$ for $\mathrm{Zr}, \mathrm{Te}_{d}$, and $\mathrm{Te}_{a}$, and $0.017 \AA$ for $\mathrm{Te}_{z}$. The band structure of $\mathrm{ZrTe}_{5}$ along $Z-\Gamma-Y$ for different displacements $\lambda$ is shown in Fig. 4. First, without any atomic displacements from equilibrium $(\lambda=0.0)$ [Fig. 4(b)], $\mathrm{ZrTe}_{5}$ has a narrow band gap of $0.04 \mathrm{eV}$. Both the valence- and conduction-band edges appear along $\Gamma-Y$ and are off the $\Gamma$ point toward the $Y$ point. The projection (green shadow) on the $\mathrm{Te}_{d} p$ orbitals clearly shows the band inversion between the valence and conduction bands, which agrees with the gapped massless Dirac state observed in experiments [13]. As one of the indicators, the 2D topological index on the $k_{z}=0$ plane is 1 [see Fig. S3(a) in the Supplemental Material [24]] shown by the odd number of crossings for the Wannier charge centers (WCCs) moving along $k_{y}$. The overall topological invariant index is $(1 ; 110)$ for the initial state. For $\lambda=-3.0$ [Fig. 4(a)], the band gap increases to $0.07 \mathrm{eV}$, but the band inversion along the $Z-$ $\Gamma-Y$ directions remains. The system is still a gapped massless Dirac state [33]. Most interestingly, in contrast to the above, by moving in the other direction with more positive $\lambda$, the band gap decreases. The valence and conduction bands touch at the $\Gamma$ point when $\lambda=2.15$ [Fig. 4(c)]. Then, the band gap reopens as $\lambda$ increases further. For $\lambda=3.0$ [Fig. 4(d)], the reopened band gap has no band inversion along $Z-\Gamma-Y$, as seen from the orbital projection of the $\mathrm{Te}_{d} p$ orbitals (inset, green line). The corresponding 2D topological index on the $k_{z}=0$ plane is now 0 , shown by the even number of crossings for the WCCs moving along $k_{y}$ in Fig. S3(b) of the Supplemental Material [24]. The overall topological index becomes $(0 ; 110)$ for a WTI. Thus, with the coherently excited Raman $A_{1 g}$ mode within one THzpulse cycle above threshold $E_{\mathrm{th}}, \mathrm{ZrTe}_{5}$ can be driven into coherent topological oscillations between the STI and WTI states, with an interesting critical Dirac point in between. The dephasing of the phonon vibration from
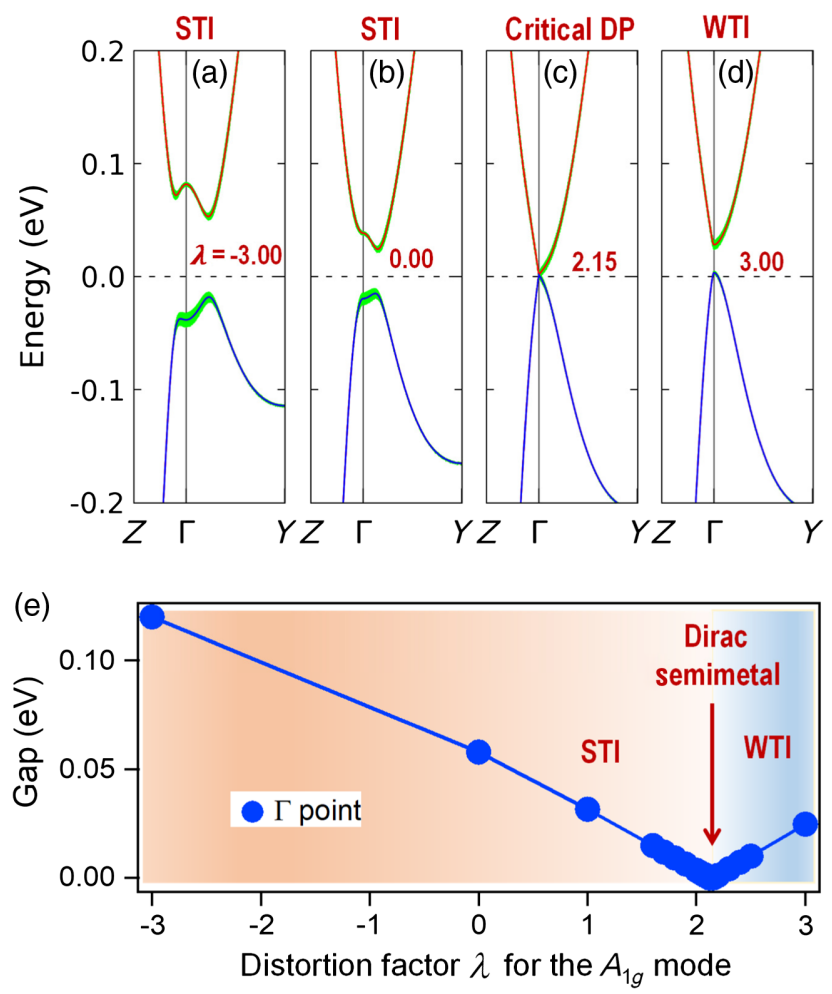

FIG. 4. Electronic band structure calculations for the topological phase switching via excitation of the $A_{1 g}$ phonon mode in $\mathrm{ZrTe}_{5}$. The band structure along $Z-\Gamma-Y$ for different magnitudes of the lattice distortion factor $\lambda$ corresponding to the $A_{1 g}$ mode [see Fig. 1(c)]: (a) -3.00, (b) 0.00, (c) 2.15, and (d) 3.00. For $\lambda=1.0$, the corresponding atomic displacements for the $A_{1 g}$ mode are $0.033 \AA$ for $\mathrm{Zr}, \mathrm{Te}_{d}$, and $\mathrm{Te}_{a}$, and $0.017 \AA$ for $\mathrm{Te}_{z}$. The green shadow indicates the projection of $\mathrm{Te}_{d} p$ orbitals denoting band inversion. This procedure identifies the states with $\lambda=-3.00,0.00$ (a),(b) as STI having a topological index of $(1 ; 110)$, whereas the critical Dirac point occurs for $\lambda=2.15$ (c). Absence of band inversion for $\lambda=3.00$ (d) indicates a WTI with the topological index $(0 ; 110)$. (e) Energy gap at the $\Gamma$ point as calculated from the band structure as a function of the atomic displacement magnitudes $\lambda$.

multiple Dirac fermion-phonon scattering and/or disorder effects results in nonthermal phonon populations, which lead to a renormalized spectral shape different from thermal ones shown in Fig. 3(a). This critical bulk DP point at $\lambda=2.15$ is marked by the vertical arrow in the $\lambda$ dependence of the band gap [Fig. 4(e)], which can exist at the $\Gamma$ point in $\mathrm{ZrTe}_{5}$ as the phase boundary between the gapped Dirac state and WTI. Such a transition can be driven by the $A_{1 g}$ Raman phonon mode consistent with the measured THz-driven states. We note that a recent study reported a direct measurement of the atomic displacement of a low-frequency shear phonon mode vs THz-field strength in a Weyl semimetal [9]. Comparing the two excitation conditions, we can estimate an atomic displacement of approximately $0.07 \AA$ for the $750-\mathrm{kV} / \mathrm{cm}$ field and is on the same order of magnitude 
needed for driving the topological phase transition by the $A_{1 g}$ mode at the critical Dirac point $(\lambda=2.15)$ in the simulation.

It is also important to note that unlike the low-energy $\mathrm{THz}$ excitation, the huge mismatch between the optical pump vs $A_{1 g}$ mode energy may lead to many spurious effects and mask the intrinsic Dirac dynamics, e.g., the excitation of topological trivial bands, incoherent electron and phonon heating, and more rapid loss of coherence that inhibits the formation of the metastable state. In addition, we also theoretically examine the other modes such as $B_{2 g}$ excited in the driven state [Fig. 1(b)]. Unlike the $A_{1 g}$ mode involving the lattice distortion along the stacking $b$ axis [Fig. 1(c)], the $B_{2 g}$ mode at $2.16 \mathrm{THz}$ involves atomic displacements of Te along the $a$ axis, a torsion of the Te dimer, and thereby costs higher energy, i.e., not as effective as the $A_{1 g}$ mode to change the band inversion between the $p$ orbitals of the $\mathrm{Te}_{d}$ and $\mathrm{Te}_{z}$ atoms.

\section{CONCLUSION}

In summary, we identify a previously inaccessible topological tuning scheme via mode-selective Raman coherence. We implement it by demonstrating THz-driven topological phase transition during coherent lattice oscillations in a Dirac semimetal. This topological quantum switching dynamics warrant further investigation to resolve them through direct electronic structure probes within the phonon oscillation cycle. This dynamic lattice-driven scheme may also be extended to diverse complex materials and driven phenomena, such as symmetry-breaking transitions to induce Weyl states [34], magnetic materials [35,36], photovoltaic semiconductors [22,37], and superconductors $[38,39]$.

\section{ACKNOWLEDGMENTS}

This work is supported by the U.S. Department of Energy, Office of Basic Energy Science, Division of Materials Sciences and Engineering (Ames Laboratory is operated for the U.S. Department of Energy by Iowa State University under Contract No. DE-AC02-07CH11358) (science driver, $\mathrm{THz}$ and Raman spectroscopy, and data analysis). Sample development and transport measurements at Brookhaven National Laboratory (Q. L., P. M. L., and G. G.) are supported by the U.S. Department of Energy, Office of Basic Energy Science, Materials Sciences and Engineering Division, under Contract No. DE-SC0012704. L.-L. W. is supported by the Center for the Advancement of Topological Semimetals, an Energy Frontier Research Center funded by the U.S. Department of Energy, Office of Basic Energy Sciences (DFT calculation and topological index analysis). M.M. and I.E.P. at the University of Alabama, Birmingham are supported by the U.S. Department of Energy, Office of Science, Basic Energy Sciences under Award No. DE-SC0019137 (THz data analysis). The THz Instrument is supported in part by National Science Foundation Grant No. EECS 1611454.

[1] D. N. Basov, R. D. Averitt, and D. Hsieh, Towards Properties on Demand in Quantum Materials, Nat. Mater. 16, 1077 (2017).

[2] T. Li, A. Patz, L. Mouchliadis, J. Yan, T. A. Lograsso, I. E. Perakis, and J. Wang, Femtosecond Switching of Magnetism via Strongly Correlated Spin-Charge Quantum Excitations, Nature (London) 496, 69 (2013).

[3] D. Kharzeev and Q. Li, The Chiral Qubit: Quantum Computing with Chiral Anomaly, arXiv:1903.07133

[4] L. Luo, X. Yang, X. Liu, Z. Liu, C. Vaswani, D. Cheng, M. Mootz, X. Zhao, Y. Yao, C.-Z. Wang, K.-M. Ho, I. E. Perakis, M. Dobrowolska, J. K. Furdyna, and J. Wang, Ultrafast Manipulation of Topologically Enhanced Surface Transport Driven by Mid-Infrared and Terahertz Pulses in $\mathrm{Bi}_{2} \mathrm{Se}_{3}$, Nat. Commun. 10, 607 (2019).

[5] N. Sirica et al., Tracking Ultrafast Photocurrents in the Weyl Semimetal TaAs Using THz Emission Spectroscopy, Phys. Rev. Lett. 122, 197401 (2019).

[6] C. Vaswani et al., Discovery of Terahertz Second Harmonic Generation from Lightwave Acceleration of SymmetryBreaking Nonlinear Supercurrents, arXiv:1912.01676.

[7] H. Huebener, M. A. Sentef, U. d. Giovannini, A. F. Kemper, and A. Rubio, Creating Stable Floquet-Weyl Semimetals by Laser-Driving of $3 D$ Dirac Materials, Nat. Commun. 8, 13940 (2017).

[8] X. Yang et al., Light Control of Surface-Bulk Coupling by Terahertz, Vibrational Coherence in a Topological Insulator, npj Quantum Mater. 5, 13 (2020).

[9] E. Sie et al., An Ultrafast Symmetry Switch in a Weyl Semimetal, Nature (London) 565, 61 (2019).

[10] D. Fausti, R. I. Tobey, N. Dean, S. Kaiser, A. Dienst, M. C. Hoffmann, S. Pyon, T. Takayama, H. Takagi, and A. Cavalleri, Light-Induced Superconductivity in a StripeOrdered Cuprate, Science 331, 189 (2011).

[11] M. Kozina, M. Fechner, P. Marsik, T. van Driel, J. M. Glownia, C. Bernhard, M. Radovic, D. Zhu, S. Bonetti, U. Staub, and M. C. Hoffmann, Terahertz-Driven Phonon Upconversion in $\mathrm{SrTiO}_{3}$, Nat. Phys. 15, 387 (2019).

[12] X. Li, T. Qiu, J. Zhang, E. Baldini, J. Lu, A. M. Rappe, and K. A. Nelson, Terahertz Field-Induced Ferroelectricity in Quantum Paraelectric $\mathrm{SrTiO}_{3}$, Science 364, 1079 (2019).

[13] Q. Li, D. E. Kharzeev, C. Zhang, Y. Huang, I. Pletikosić, A. V. Fedorov, R. D. Zhong, J. A. Schneeloch, G. D. Gu, and T. Valla, Chiral Magnetic Effect in $\mathrm{ZrTe}_{5}$, Nat. Phys. 12, 550 (2016).

[14] H. Weng, X. Dai, and Z. Fang, Transition-Metal Pentatelluride $\mathrm{ZrTe}_{5}$ and $\mathrm{HfTe}_{5}$ : A Paradigm for Large-Gap Quantum Spin Hall Insulators, Phys. Rev. X 4, 011002 (2014).

[15] F. Tang et al., Three-Dimensional Quantum Hall Effect and Metal-Insulator Transition in $\mathrm{ZrTe}_{5}$, Nature (London) 569 , 537 (2019).

[16] B. Xu, L. X. Zhao, P. Marsik, E. Sheveleva, F. Lyzwa, Y. M. Dai, G. F. Chen, X. G. Qiu, and C. Bernhard, TemperatureDriven Topological Phase Transition and Intermediate 
Dirac Semimetal Phase in $\mathrm{ZrTe}_{5}$, Phys. Rev. Lett. 121, 187401 (2018).

[17] J. Mutch, W.-C. Chen, P. Went, T. Qian, I. Z. Wilson, A. Andreev, C.-C. Chen, and J.-H. Chu, Evidence for a Strain Tuned Topological Phase Transition in $\mathrm{ZrTe}_{5}$, Sci. Adv. 5, eaav9771 (2019).

[18] Z.-G. Chen, R. Y. Chen, R. D. Zhong, J. Schneeloch, C. Zhang, Y. Huang, F. Qu, R. Yu, Q. Li, G. D. Gu, and N. L. Wang, Optical Spectroscopy Study of the Three-Dimensional Dirac Semimetal $\mathrm{ZrTe}_{5}$, Proc. Natl. Acad. Sci. U.S.A. 114, 819 (2017).

[19] H. Chi, C. Zhang, G. Gu, D. E. Kharzeev, X. Dai, and Q. Li, Lifshitz Transition Mediated Electronic Transport Anomaly in Bulk $\mathrm{ZrTe}_{5}$, New J. Phys. 19, 015005 (2017).

[20] X. Yang et al., Terahertz-Light Quantum Tuning of a Metastable Emergent Phase Hidden by Superconductivity, Nat. Mater. 17, 586 (2018).

[21] X. Yang, C. Vaswani, C. Sundahl, M. Mootz, L. Luo, J. H. Kang, I. E. Perakis, C. B. Eom, and J. Wang, LightwaveDriven Gapless Superconductivity and Forbidden Quantum Beats by Terahertz Symmetry Breaking, Nat. Photonics 13, 707 (2019).

[22] Z. Liu et al., Single-Cycle Terahertz Driven Quantum Beats Reveal Symmetry-Selective Control of Excitonic Fine Structure in Perovskite, arXiv:1905.12373.

[23] X. Yang, L. Luo, M. Mootz, A. Patz, S. L. Budko, P. C. Canfield, I. E. Perakis, and J. Wang, Non-Equilibrium Pair Breaking in $\mathrm{Ba}\left(\mathrm{Fe}_{1-x} \mathrm{Co}_{x}\right)_{2} \mathrm{As}_{2}$ Superconductors: Evidence for Formation of Photo-Induced Excitonic Spin-DensityWave State, Phys. Rev. Lett. 121, 267001 (2018).

[24] See Supplemental Material at http://link.aps.org/ supplemental/10.1103/PhysRevX.10.021013 for sample properties (Sec. 1), transport measurements (Sec. 2), THz transmission spectra (Sec. 3), and additional DFT calculations (Sec. 4).

[25] T. Liang et al., Anomalous Hall Effect in $\mathrm{ZrTe}_{5}$, Nat. Phys. 14, 451 (2018).

[26] D. M. Juraschek and S. F. Maehrlein, Sum-Frequency Ionic Raman Scattering, Phys. Rev. B 97, 174302 (2018).

[27] G. Manzoni et al., Ultrafast Optical Control of the Electronic Properties of $\mathrm{ZrTe}_{5}$, Phys. Rev. Lett. 115, 207402 (2015).

[28] X. Zhang et al., Ultrafast Hot Carrier Dynamics of $\mathrm{ZrTe}_{5}$ from Time-Resolved Optical Reflectivity, Phys. Rev. B 99, 125141 (2019).
[29] See, e.g., T. Li, L. Luo, M. Hupalo, J. Zhang, M. C. Tringides, J. Schmalian, and J. Wang, Femtosecond Population Inversion and Stimulated Emission of Dense Dirac Fermions in Graphene, Phys. Rev. Lett. 108, 167401 (2012), and references therein.

[30] K. Sumida et al., Prolonged Duration of Nonequilibrated Dirac Fermions in Neutral Topological Insulators, Sci. Rep. 7, 14080 (2017).

[31] A. Zong et al., Dynamical Slowing-Down in an Ultrafast Photoinduced Phase Transition, Phys. Rev. Lett. 123, 097601 (2019).

[32] G. Landa, A. Zwick, R. Carles, M. A. Renucci, and A. Kjekshus, Lattice-Dynamics of the Transition-Metal Pentatellurides $\mathrm{ZrTe}_{5}$ and $\mathrm{HfTe}_{5}$, Solid State Commun. 50, 297 (1984).

[33] C. J. Tabert, J. P. Carbotte, and E. J. Nicol, Optical and Transport Properties in Three-Dimensional Dirac and Weyl Semimetals, Phys. Rev. B 93, 085426 (2016).

[34] L.-L. Wang, N. H. Jo, Y. Wu, Q. S. Wu, A. Kaminski, P. C. Canfield, and D. D. Johnson, Phonon-Induced Topological Transition to a Type-II Weyl Semimetal, Phys. Rev. B 95, 165114 (2017).

[35] A. Patz, T. Li, S. Ran, R. M. Fernandes, J. Schmalian, S. L. Bud'ko, P. C. Canfield, I. E. Perakis, and J. Wang, Ultrafast Observation of Critical Nematic Fluctuations and Giant Magnetoelastic Coupling in Iron Pnictides, Nat. Commun. 5, 3229 (2014).

[36] A. Patz, T. Li, X. Liu, J. K. Furdyna, I. E. Perakis, and J. Wang, Ultrafast Probes of Nonequilibrium Hole Spin Relaxation in the Ferromagnetic Semiconductor GaMnAs, Phys. Rev. B 91, 155108 (2015).

[37] L. Luo, L. Men, Z. Liu, Y. Mudryk, X. Zhao, Y. Yao, J.-M. Park, R. Shinar, J. Shinar, K.-M. Ho, I. E. Perakis, J. Vela, and J. Wang, Ultrafast Terahertz Snapshots of Eexcitonic Rydberg States and Electronic Coherence in an Organometal Halide Perovskite, Nat. Commun. 8, 15565 (2017).

[38] X. Yang et al., Ultrafast Nonthermal Terahertz Electrodynamics and Possible Quantum Energy Transfer in the $\mathrm{Nb}_{3} \mathrm{Sn}$ Superconductor, Phys. Rev. B 99, 094504 (2019).

[39] A. Patz, T. Li, L. Luo, X. Yang, S. Bud'ko, P. C. Canfield, I. E. Perakis, and J. Wang, Critical Speeding up of Nonequilibrium Electronic Relaxation near Nematic Phase Transition in Unstrained $\mathrm{Ba}\left(\mathrm{Fe}_{1-x} \mathrm{Co}_{x}\right)_{2} \mathrm{As}_{2}$, Phys. Rev. B 95, 165122 (2017). 